\title{
Quantum and classical nonlinear dynamics in a microwave cavity
}

\author{
Charles H Meaney ${ }^{1}$, Hyunchul Nha ${ }^{2}$, Timothy Duty ${ }^{3}$ and Gerard J Milburn ${ }^{1 *}$
}

${ }^{*}$ Correspondence:

milburn@physics.uq.edu.au

'Department of Physics, The

University of Queensland, St Lucia,

QLD 4072, Australia

Full list of author information is

available at the end of the article

\begin{abstract}
We consider a quarter wave coplanar microwave cavity terminated to ground via a superconducting quantum interference device. By modulating the flux through the loop, the cavity frequency is modulated. The flux is varied at twice the cavity frequency implementing a parametric driving of the cavity field. The cavity field also exhibits a large effective nonlinear susceptibility modelled as an effective Kerr nonlinearity, and is also driven by a detuned linear drive. We show that the semi-classical model corresponding to this system exhibits a fixed point bifurcation at a particular threshold of parametric pumping power. We show the quantum signature of this bifurcation in the dissipative quantum system. We further linearise about the below threshold classical steady state and consider it to act as a bifurcation amplifier, calculating gain and noise spectra for the corresponding small signal regime. Furthermore, we use a phase space technique to analytically solve for the exact quantum steady state. We use this solution to calculate the exact small signal gain of the amplifier.
\end{abstract}

Keywords: superconducting circuit; parametric amplifier; quantum noise

\section{Introduction}

Superconducting circuit quantum electrodynamics (circuit QED) [1] is increasingly being used to study systems in the quantum regime. This experimental context sees a superconducting coplanar waveguide act as a microwave cavity, in contrast to the optical frequency cavities of traditional cavity quantum electrodynamics (cavity QED). The microwave resonator is made from aluminium on a silicon substrate, and Josephson junctions are created by allowing the aluminium to oxidise before adding more aluminium. Such devices are placed in a dilution refrigerator, and experiments take place at cryogenic temperatures. Such low temperatures, close to the quantum ground state, allow quantum mechanical phenomena to become manifest. Recent engineering progress means that fabrication of these devices is possible [2].

In recent experiments at Chalmers [3], a quarter wave coplanar microwave cavity is terminated to ground via one or more superconducting quantum interference devices (SQUIDs), see Figure 1. By modulating the flux through the loop, the cavity frequency can be modulated. If the flux is varied at twice the cavity frequency this implements a parametric driving of the cavity field. The cavity field also exhibits a large effective nonlinear susceptibility that can be modelled as an intensity dependent phase shift [4].

(c) 2014 Meaney et al.; licensee Springer on behalf of EPJ. This is an Open Access article distributed under the terms of the Creative Commons Attribution License (http://creativecommons.org/licenses/by/2.0), which permits unrestricted use, distribution, and reproduction in any medium, provided the original work is properly cited. 
Figure 1 Schematic of the system under consideration in this paper. A schematic of the system under consideration. A superconducting microwave cavity of frequency $\omega_{c}$ has an effective Kerr nonlinearity $\chi$, due primarily to the SQUID-loop connecting it to ground. It is driven both by a linear drive of amplitude $\epsilon$ at a detuning from the cavity of $\Delta$, and also by a parametric drive at twice the cavity frequency at an amplitude $\kappa$.

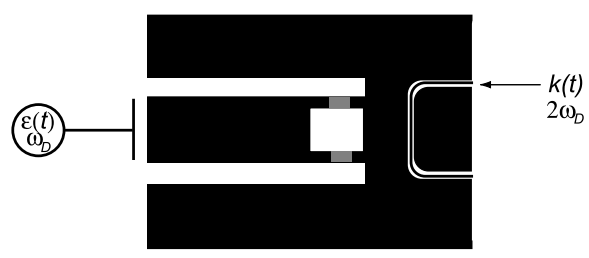

This paper is structured as follows. In Section 2 we introduce the nonlinear microwave system considered in this paper. We establish a description in the form of a Markov master equation, one term of which being an effective Hamiltonian we derive. We also give the input-output formulation of the microwave system. In Section 3 we present a detailed analysis of the fixed point structure of the nonlinear microwave system in a semi-classical description, including bifurcation of the fixed points. We include dissipation of the microwave mode. In Section 4 we look at the steady state of the quantum system. This is done in a phase space representation based on the positive P-representation, both analytically, and numerically. We look for signatures of the semi-classical bifurcations. In Section 5 we analytically compute and plot the small signal gain. Then, in Section 6 we linearise the model and extract gain and noise spectra up to the threshold defined by the semi-classical bifurcation. Finally in Section 7 we summarise our results.

A similar model to that considered here has been given by Wustmann and Shumeiko [5]. Their discussion of the semiclassical steady states and fixed point structure parallels the discussion here but gives a more detailed description of the semiclassical dynamics. They also discuss the quantum noise features of the model using a linearised quantum Langevin approach. In addition to a linearised analysis of the gain and signal-to-noise ratio, we give an exact steady state solution for the quantum master equation using the positive P-representation. The steady state behaviour of the model we describe has been experimentally observed by Wilson et al. [6].

\section{The dissipative Cassinian oscillator model}

\subsection{Master equation}

We consider a superconducting microwave resonator connected through a superconducting quantum interference device (SQUID) loop to ground. The SQUID loop consists of two separated Josephson junctions; a magnetic flux can then be applied to loop to change the effective resonant frequency of the cavity [3]. The SQUID also induces a significant quartic nonlinearity. Following Wallquist et al. [4] we describe the integrated cavity + SQUID system in terms of an equivalent circuit composed of a capacitor and a nonlinear inductor. The Hamiltonian for one mode of the cavity field may be written in terms of this effective nonlinear oscillator as [4]

$$
\mathcal{H}=E_{C} n^{2}+E_{L} \phi^{2}+\lambda \phi^{4}
$$

where $E_{C}=\frac{(2 e)^{2}}{2 C}$ represents the charging energy of the effective LC oscillator while $n$ is the number of elementary charges on the capacitor, $E_{L}=\frac{\hbar^{2}}{2(2 e)^{2} L}$ represents the inductive energy of the effective oscillator, while $\phi$ represents the flux though the equivalent inductor and $\lambda$ represents the inductive nonlinearity. This depends on the inductive energy scale 
and on the mode function of the cavity field $\lambda=E_{L} B$, where $B$ is a geometric factor. Further details are given in Wallquist et al. give [4].

The system may be quantised by introducing the bosonic raising and lowering operators, defined in terms of the canonical variables of the cavity field as,

$$
\begin{aligned}
& \hat{\phi}=\left(\frac{E_{C}}{4 E_{L}}\right)^{\frac{1}{4}}\left(\hat{a}+\hat{a}^{\dagger}\right), \\
& \hat{n}=-\mathrm{i}\left(\frac{E_{L}}{4 E_{C}}\right)^{\frac{1}{4}}\left(\hat{a}-\hat{a}^{\dagger}\right),
\end{aligned}
$$

where $\hat{\psi}$ and $\hat{n}$ are the average phase across the junctions and charge on the junctions, respectively and $C$ and $L$ are the effective lumped capacitance and inductance, respectively, of the equivalent circuit for the cavity in terms of which the cavity resonant frequency is give by $\omega_{c}=\frac{1}{\sqrt{L C}}$. The total Hamiltonian, including the coherent driving and the parametric driving are then given by

$$
\mathcal{H}=\hbar \omega_{c} \hat{a}^{\dagger} \hat{a}+\hbar\left(\epsilon^{*} \hat{a} \mathrm{e}^{\mathrm{i} \omega_{D} t}+\epsilon \hat{a}^{\dagger} \mathrm{e}^{-\mathrm{i} \omega_{D} t}\right)+\hbar \chi \hat{a}^{\dagger} \hat{a}^{2}+\frac{\hbar}{2}\left(\kappa^{*} \hat{a}^{2} \mathrm{e}^{\mathrm{i} 2 \omega_{D} t}+\kappa \hat{a}^{\dagger} \mathrm{e}^{-\mathrm{i} 2 \omega_{D} t}\right),
$$

where $\omega_{c}$ is the cavity frequency, $\epsilon=|\epsilon| \mathrm{e}^{\mathrm{i} v}$ represents the coherent driving strength, $\kappa$ represents the parametric driving strength, $\omega_{D}$ is the coherent driving frequency and we have assumed that the parametric driving is at twice the coherent driving frequency and $v$ is the phase difference between the coherent driving and the parametric driving as we have taken the phase of the parametric driving term as zero. In Section 6 we consider coherent homodyne detection of the cavity output. This means there is another phase in this problem; the phase choice for the local oscillator which may not be in phase with either the coherent or the parametric driving. The term proportional to $\chi$ represents a nonlinear (quartic) phase shift that arises from the nonlinear inductance of the SQUID loop. Quartic non-linearities in oscillators have been discussed in $[7,8]$; parametric terms in the nano-electromechanical context have been discussed in [9-11].

For a realistic device we adopt a dissipative model. We model the microwave cavity resonator as being damped in a zero temperature heat bath. Such a model for the bath is certainly justified as the typical microwave cavity is at $\mathrm{mK}$ temperature and thus the mean excitation photon number is very close to zero [12]. The amplitude decay rate for the microwave cavity is $\gamma$. We then describe the dissipative dynamics with the master equation (with weak damping and the rotating wave approximation for the system-environment couplings). In an interaction picture at the coherent driving frequency this is

$$
\frac{\mathrm{d} \hat{\rho}}{\mathrm{d} t}=-\frac{\mathrm{i}}{\hbar}[\hat{\mathcal{H}}, \hat{\rho}]+\gamma\left(2 \hat{a} \hat{\rho} \hat{a}^{\dagger}-\hat{a}^{\dagger} \hat{a} \hat{\rho}-\hat{\rho} \hat{a}^{\dagger} \hat{a}\right),
$$

where $\hat{\rho}$ is the density matrix of the microwave cavity, and $\hat{\mathcal{H}}$ is the Hamiltonian in an interaction picture in a rotating frame with respect to the linear driving frequency. We have made the rotating wave approximation by ignoring terms with frequency $2 \omega_{D}$ or above. We thus have

$$
\hat{\mathcal{H}}=\hbar \Delta \hat{a}^{\dagger} \hat{a}+\hbar\left(\epsilon^{*} \hat{a}+\epsilon \hat{a}^{\dagger}\right)+\frac{\hbar}{2}\left(\kappa^{*} \hat{a}^{2}+\kappa \hat{a}^{\dagger 2}\right)+\frac{\hbar \chi}{2} \hat{a}^{\dagger} \hat{a}^{2},
$$


where $\Delta=\omega_{c}-\omega_{D}$. In the absence of damping, classical trajectories arising from the parametric and nonlinear portion of this Hamiltonian are the ovals of Cassini, and that system is hence sometimes described as the 'Cassinian' oscillator; the quantum version of that part of the Hamiltonian system has been previously studied by Wielinga et al. [13] and more recently by Dykman and his collaborators [14].

The semi-classical dynamics and the exact quantum steady state can be found using the complex P-function of Drummond and Gardiner [15]. In this approach the density operator in terms of the off diagonal projectors onto oscillator coherent states

$$
\hat{\rho}(t)=\int \mathrm{d} \alpha \mathrm{d} \beta P(\alpha, \beta, t) \frac{|\alpha\rangle\left\langle\beta^{*}\right|}{\left\langle\beta^{*} \mid \alpha\right\rangle} .
$$

This function determines the normally ordered moments by

$$
\left\langle\hat{a}^{\dagger m} \hat{a}^{n}\right\rangle=\int \mathrm{d} \alpha \mathrm{d} \beta P(\alpha, \beta, t) \alpha^{n} \beta^{m} .
$$

It may seem surprising at first sight to notice that the Positive P-function has support in a phase space with twice as many canonical variables as the corresponding classical problem. There is a direct physical interpretation of the extra variables based on a measurement model in which there are twice as many readout channels for the canonical phase space variable [16]. This is required if the distributions are to give normally ordered moments directly via integration. In [17] a direct implementation using circuit QED of these additional channels is demonstrated and connection is made to the stationary normal ordered moments.

The master equation can then be converted into a Fokker-Planck like equation for the P-function,

$$
\begin{aligned}
\frac{\partial P(\alpha, \beta)}{\partial t}= & \left\{\partial_{\alpha}\left[(\gamma+\mathrm{i} \Delta) \alpha+\mathrm{i} \epsilon+\mathrm{i} \chi \beta \alpha^{2}+\mathrm{i} \kappa \beta\right]+\partial_{\beta}\left[(\gamma-\mathrm{i} \Delta) \beta-\mathrm{i} \epsilon^{*}-\mathrm{i} \chi \alpha \beta^{2}-\mathrm{i} \kappa^{*} \alpha\right]\right. \\
& \left.+\partial_{\alpha \alpha}^{2}\left[-\mathrm{i} \frac{1}{2}\left(\kappa+\chi \alpha^{2}\right)\right]+\partial_{\beta \beta}^{2}\left[\mathrm{i} \frac{1}{2}\left(\kappa^{*}+\chi \beta^{2}\right)\right]\right\}
\end{aligned}
$$

where $\partial_{\alpha}=\frac{\partial}{\partial \alpha}$ and $\partial_{\alpha \alpha}^{2}=\frac{\partial^{2}}{\partial \alpha \partial \alpha}$ etc. The corresponding stochastic differential equations are

$$
\begin{aligned}
& \mathrm{d} \alpha=-(\gamma+\mathrm{i} \Delta) \alpha \mathrm{d} t-\mathrm{i} \epsilon \mathrm{d} t-\mathrm{i}\left(\chi \alpha^{2}+\kappa\right) \beta \mathrm{d} t+\left[-\mathrm{i}\left(\kappa+\chi \alpha^{2}\right)\right]^{\frac{1}{2}} \mathrm{~d} z_{1}, \\
& \mathrm{~d} \beta=-(\gamma-\mathrm{i} \Delta) \beta \mathrm{d} t+\mathrm{i} \epsilon^{*} \mathrm{~d} t+\mathrm{i}\left(\chi \beta^{2}+\kappa^{*}\right) \alpha \mathrm{d} t+\left[\mathrm{i}\left(\kappa^{*}+\chi \beta^{2}\right)\right]^{\frac{1}{2}} \mathrm{~d} z_{2} .
\end{aligned}
$$

The semi-classical equations are obtained by setting $\beta=\alpha^{*}$ in the drift term and ignoring the diffusion term, and are thus

$$
\frac{\mathrm{d} \alpha}{\mathrm{d} t}=-\left(\gamma+\mathrm{i} \Delta+\mathrm{i} \chi|\alpha|^{2}\right) \alpha-\mathrm{i} \epsilon-\mathrm{i} \kappa \alpha^{*}
$$

from which it is apparent that the nonlinearity appears as a nonlinear detuning. This ensures that the instability in the $\chi=0$ model when $\kappa=\gamma$ does not arise. 


\section{Semi-classical fixed point structure}

\subsection{No coherent driving, $\epsilon=0$}

We first consider the case of no driving field $\epsilon=0$. The semi-classical equations of motion (10) are then given by

$$
\frac{\mathrm{d} \alpha}{\mathrm{d} t}=-(\gamma+\mathrm{i} \Delta) \alpha-\mathrm{i} \chi \alpha|\alpha|^{2}-\mathrm{i} \kappa \alpha^{*} .
$$

The semi-classical steady states, $\alpha_{0}=\sqrt{n_{0}} \mathrm{e}^{\mathrm{i} \theta_{0}}$, are given by $\bar{n}_{0}=0$, and

$$
\begin{aligned}
& \bar{n}_{0}=-\Delta^{\prime} \pm \sqrt{\left(\kappa^{\prime}\right)^{2}-1}, \\
& \sin \left(2 \theta_{0}\right)=-\frac{1}{\kappa^{\prime}},
\end{aligned}
$$

where we have defined the scaled variables

$$
\begin{aligned}
\bar{n}_{0} & =\frac{\chi}{\gamma} n_{0}, \\
\kappa^{\prime} & =\frac{\kappa}{\gamma}, \\
\Delta^{\prime} & =\frac{\Delta}{\gamma} .
\end{aligned}
$$

In order to determine the stability of the fixed points, we linearise the equations of motion around the fixed points. Thus we have the semi-classical linearised equation of motion for $\delta \alpha=\alpha-\alpha_{0}$ and $\delta \alpha^{*}=\alpha^{*}-\alpha_{0}^{*}$

$$
\left[\begin{array}{c}
\frac{\mathrm{d}(\delta \alpha)}{\mathrm{d} t} \\
\frac{\mathrm{d}\left(\delta \alpha^{*}\right)}{\mathrm{d} t}
\end{array}\right] \approx \mathbf{M}_{\alpha \alpha^{*}}\left[\begin{array}{c}
\delta \alpha \\
\delta \alpha^{*}
\end{array}\right]
$$

where

$$
\mathbf{M}_{\alpha \alpha^{*}}=\left[\begin{array}{cc}
-\gamma-\mathrm{i} \Delta-\mathrm{i} 2 \chi\left|\alpha_{0}\right|^{2} & -\mathrm{i} \chi \alpha_{0}^{2}-\mathrm{i} \kappa \\
\mathrm{i} \chi\left(\alpha_{0}^{*}\right)^{2}+\mathrm{i} \kappa & -\gamma+\mathrm{i} \Delta+\mathrm{i} 2 \chi\left|\alpha_{0}\right|^{2}
\end{array}\right] .
$$

In the limit of no parametric pumping $(\kappa=0)$, this Jacobian matches the result obtained by Babourina-Brooks et al. in [8]. Stability of the fixed point requires all the eigenvalues of the Jacobian to have a real part less than or equal to zero [18]. A real part of exactly zero indicates marginal stability in that parameter direction, where the fixed point is neither attractive nor repulsive. Real parts strictly less than zero are attracting fixed points which draw in nearby regions in phase space. In general, stability may depend on more coupling parameter combinations than those which define the fixed points.

The origin is a fixed point for all parameter values. Indeed, the origin is the only fixed point for $\kappa<\gamma$, the 'below threshold' regime. This fixed point is stable for $\kappa^{2}<\gamma^{2}+\Delta^{2}$. Four additional fixed points occur as antipodal pairs for $\kappa>\gamma$; the 'above threshold' regime. The first additional pair of fixed points, which we will call the 'stable pair', and are given by

$$
\left(\bar{n}_{0}, 2 \theta_{0}\right)=\left(-\Delta^{\prime}+\sqrt{\left(\kappa^{\prime}\right)^{2}-1},-\pi+\operatorname{arccsc} \kappa^{\prime}\right),
$$


exists for $\Delta<\sqrt{(\kappa)^{2}-\gamma^{2}}$, and is always stable. The second additional pair of fixed points, which we will call the 'unstable pair'

$$
\left(\bar{n}_{0}, 2 \theta_{0}\right)=\left(-\Delta^{\prime}-\sqrt{\left(\kappa^{\prime}\right)^{2}-1},-\operatorname{arccsc} \kappa^{\prime}\right),
$$

exists for $\Delta<-\sqrt{(k a)^{2}-\gamma^{2}}$, and is always unstable. We note that the unstable pair of fixed points can only exist for negative detuning, and that whenever the unstable pair of fixed points exists, the first pair of fixed points exists also. Also, we note that for $\epsilon=0, \hat{a} \rightarrow-\hat{a}$ is a symmetry of the system, and thus pairs of antipodal fixed points are the expected semi-classical result. We plot the radial and angular components of the semi-classical fixed points in Figure 2. The colour in these plots shows the stability.

The bifurcating behaviour of the semi-classical steady state is depicted in the 'phase digram' of Figure 3. There are two qualitatively different transitions that can take place in

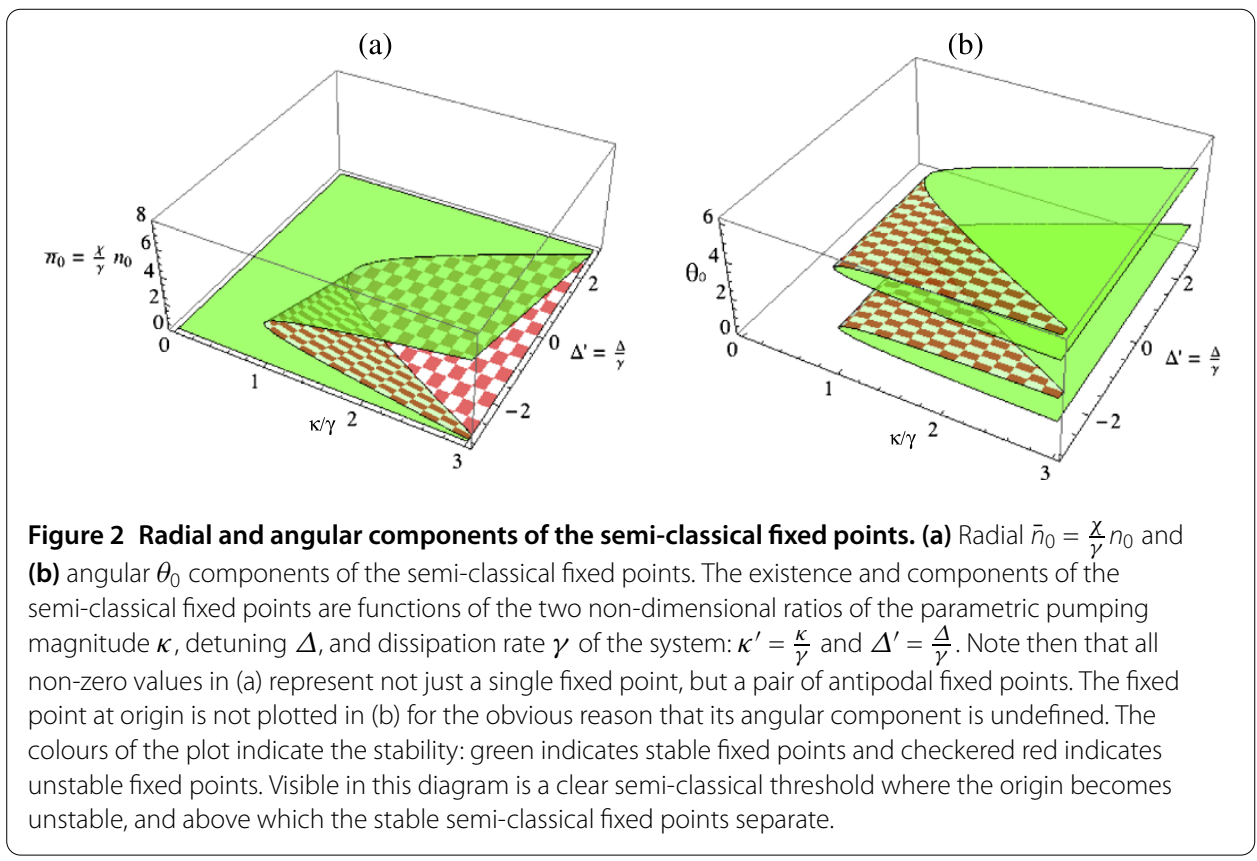

Figure 3 'Phase diagram' of the semi-classical system. The 'phase diagram' of the semi-classical system. The existence and components of the semi-classical fixed points are functions of the two non-dimensional ratios of the parametric pumping magnitude $\kappa$, detuning $\Delta$, and dissipation rate $\gamma$ of the system: $\boldsymbol{\kappa}^{\prime}=\frac{\kappa}{\gamma}$ and $\Delta^{\prime}$ $=\frac{\Delta}{\gamma}$. We also show the parameter regimes chosen for numerically computing the quantum steady state. There are three different classes of fixed points: the origin is a fixed point for all parameter values (stable in the green and striped blue regions, and unstable in the checkered red region); a stable pair of antipodal fixed points exists for 'above threshold' parametric pumping (the striped blue and checkered red regions); and an unstable pair exists for small values of 'above threshold' parametric pumping if the detuning $\Delta$ is negative (the striped blue region only). The semi-classical steady states at the specific various black circles and crosses are depicted in Figures 4 and 5 respectively. These are for comparison with the quantum steady states discussed in Section 4 and depicted in Figures 4 and 5.

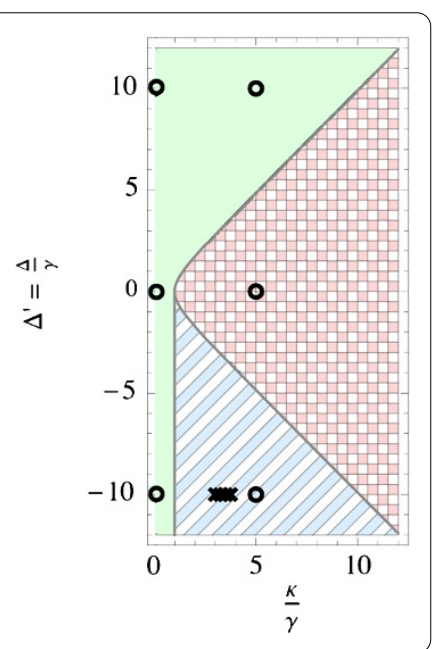


Figure 4 Semi-classical steady states of the microwave system for different regions of the phase diagram. Semi-classical steady states of the microwave system for different regions of the 'phase diagram' in Figure 3. These nine plots show the semi-classical fixed points that correspond to the parameters of the nine black circles in Figure 3. Specifically, from top-left to bottom-right, moving form left to right, then top to bottom, these parameter values are: $\left(\kappa^{\prime}, \Delta^{\prime}\right)=(0,10),(5$, $10),(0,0),(5,0),(0,-10)$, and $(5,-10)$. The colours indicate stability; the green circle markers are stable fixed points, and the red cross markers are unstable fixed points. Clearly visible are the qualitative differences in each region separated by the semi-classical bifurcations. These semi-classical fixed points can be compared with the quantum steady state Wigner functions for the same set of parameters in Figure 6.

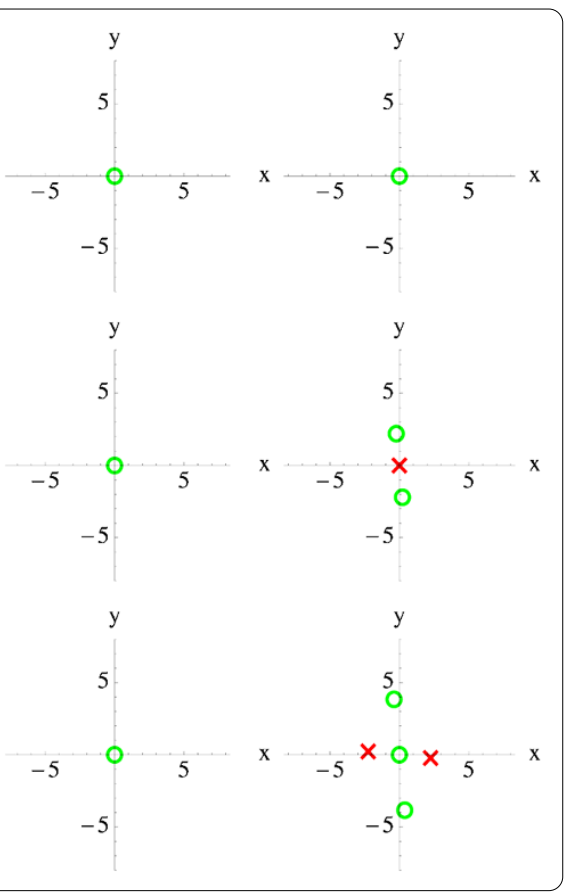

the semi-classical system as it moves from being 'below threshold' to 'above threshold' First, for positive detuning $(\Delta>0)$, the threshold parametric pumping $\kappa^{\prime}=1$ is effectively increased by the detuning to $\kappa^{\prime}=\sqrt{1+\Delta^{\prime 2}}$ (the solid green/checkered red boundary in Figure 3. At this threshold, the semi-classical system undergoes a supercritical pitchfork bifurcation where the stable origin goes unstable, and the stable pair of fixed points emerges from the origin and grows in separation with increasing parametric pumping (the checkered red region in the upper half-plane in Figure 3). Alternatively, for all values of negative detuning $(\Delta<0)$, at the threshold parametric pumping $\kappa^{\prime}=1$ two saddle-node bifurcations produce both the stable and unstable fixed point pairs (the solid green/striped blue boundary in Figure 3). The origin remains stable, and the two newly created pairs of fixed points then exist for parametric pumping above threshold $\left(\kappa^{\prime}>1\right)$ until pumping reaches the even higher value $\kappa^{\prime}=\sqrt{1+\Delta^{\prime 2}}$. Between these two values (the striped blue region in Figure 3) with increasing parametric pumping, the stable pair increases in separation and the unstable pair moves to the origin. At the higher parametric pumping $\kappa^{\prime}=\sqrt{1+\Delta^{\prime 2}}$ (the striped blue/checkered red boundary in Figure 3) the unstable pair annihilates in a subcritical pitchfork bifurcation at the origin, and the origin becomes unstable for all higher parametric pumping $\kappa^{\prime}>\sqrt{1+\Delta^{\prime 2}}$. The stable pair of fixed points continues to grow in separation with further increased parametric pumping (the checkered red region in the lower half-plane in Figure 3).

We now illustrate the steady state behaviour of the semi-classical microwave system in different 'phases'. We choose a point in parameter space from each region of the semiclassical 'phase diagram' of Figure 3. These choices are marked with the black circles in that figure. These steady states are shown in Figure 4. Some other near identical steady states, corresponding to the black crosses in Figure 3 are depicted in Figure 5. These are plotted for comparison with the quantum version of the system in Section 4.

As well as the bifurcation structure, an important observation to make at this point is that the existence and bifurcations of the fixed points depend upon only three parame- 


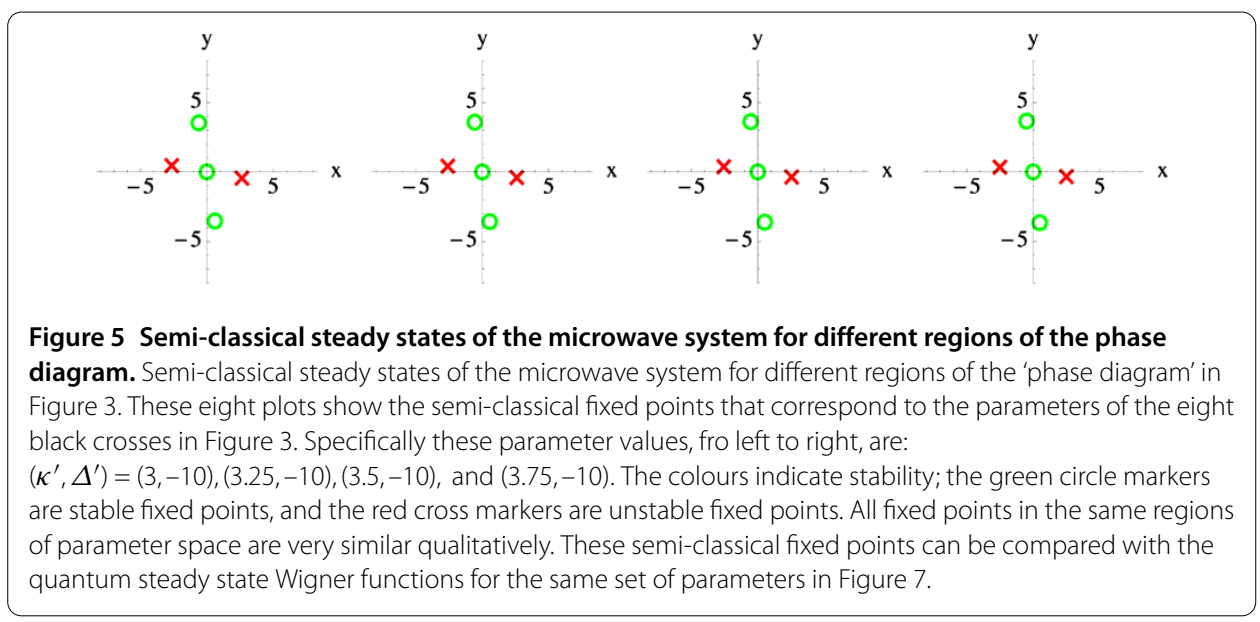

ters: the magnitude of the parametric pumping rate $\kappa$; the detuning $\Delta$; and the dissipation rate $\gamma$. And specifically, only the two non-dimensional ratios of them, here we chose $\kappa^{\prime}=\frac{\kappa}{\gamma}$ and $\Delta^{\prime}=\frac{\Delta}{\gamma}$. Thus, the below threshold to above threshold transition of the parametric oscillator is independent of the size of the induced Kerr nonlinearity $\chi$. However, the separation of the semi-classical fixed points $\sqrt{\bar{n}_{0}}=\sqrt{\frac{\chi}{\gamma} n_{0}}$, and thus the degree and visibility of the above-threshold oscillations, depends on the scaling parameter $\frac{x}{\gamma}$. Thus, to see the semi-classical fixed points move significantly away from the origin, and thus to observe significant above-threshold behaviour we require a significantly large nonlinearity $\chi$ as well as parametric pumping $\kappa$.

It will also prove useful to consider the non-dissipative limit $(\gamma \rightarrow 0)$ of the semiclassical equations on resonance $(\Delta \rightarrow 0)$. The fixed points are no longer stable zero dimensional attractors but rather elliptical fixed points corresponding to stable small oscillations in the corresponding Hamiltonian model. One easily sees that the fixed points occur at

$$
\alpha_{0}^{2}=-\frac{\kappa}{\chi}
$$

\subsection{Including coherent driving, $\epsilon \neq 0$}

Similar to our definitions $\alpha_{0}=\sqrt{\bar{n}_{0}} \mathrm{e}^{\mathrm{i} \theta_{0}}$ and $\bar{n}_{0}$, we introduce the scaled Cartesian coordinates $\bar{x}_{0}$ and $\bar{y}_{0}$ such that $\alpha_{0}=x_{0}+\mathrm{i} y_{0}$ and

$$
\begin{aligned}
& \bar{x}_{0}=\sqrt{\frac{\chi}{\gamma}} x_{0}, \\
& \bar{y}_{0}=\sqrt{\frac{\chi}{\gamma}} y_{0} .
\end{aligned}
$$

We also define the scaled linear driving

$$
\bar{\epsilon}^{\prime}=\sqrt{\frac{\chi}{\gamma}} \frac{\epsilon}{\gamma} .
$$


In terms of these, the fixed points of the semi-classical equation of motion (10) satisfy the quintic equation

$$
\begin{aligned}
0= & 4 \kappa^{\prime 2} \bar{y}_{0}^{5}-4 \Im\left\{\bar{\epsilon}^{\prime}\right\} \kappa^{\prime} \bar{y}_{0}^{4}+\left[4\left(\kappa^{\prime}+\Delta^{\prime} \kappa^{\prime 2}-\kappa^{\prime 3}\right)+\left|\bar{\epsilon}^{\prime}\right|^{2}\right] \bar{y}_{0}^{3} \\
& +2\left[\Re\left\{\bar{\epsilon}^{\prime}\right\}\left(\Delta^{\prime}-2 \kappa^{\prime}\right)+\Im\left\{\bar{\epsilon}^{\prime}\right\}\left(1+2 \kappa^{\prime}\left(\Delta^{\prime}-2 \kappa^{\prime}\right)\right)\right] \bar{y}_{0}^{2} \\
& +\left[1+\Delta^{\prime 2}-\kappa^{\prime 2}-3 \Re\left\{\bar{\epsilon}^{\prime}\right\} \Im\left\{\bar{\epsilon}^{\prime}\right\}+\Im\left\{\bar{\epsilon}^{\prime}\right\}^{2}\left(\Delta^{\prime}-5 \kappa^{\prime}\right)\right] \bar{y}_{0} \\
& +\left[\Re\left\{\bar{\epsilon}^{\prime}\right\}+\Im\left\{\bar{\epsilon}^{\prime}\right\}\left(\Delta^{\prime}+\kappa^{\prime}\right)+\Im\left\{\bar{\epsilon}^{\prime}\right\}^{3}\right] .
\end{aligned}
$$

We notice that were there to be only a small input signal and not a large linear drive with a small input signal on top $\left(\epsilon=\bar{\epsilon}^{\prime}=0\right)$, then the quintic factorises into the quadratic

$$
0=\bar{y}_{0}\left(4 \kappa^{\prime 2}\left(\bar{y}_{0}^{2}\right)^{2}+4\left(\kappa^{\prime}+\Delta^{\prime} \kappa^{\prime 2}-\kappa^{\prime 3}\right) \bar{y}_{0}^{2}+1+\Delta^{\prime 2}-\kappa^{\prime 2}\right) .
$$

This of course defines the tractable analytic fixed points given in Section 3.1.

Unfortunately, solving a quintic equation analytically in terms of radicals can lead to unhelpful expressions, and is not even always possible. We can of course numerically solve for the fixed points for certain parameter values, but we leave non-perturbative exploration of the steady states of the $\epsilon \neq 0$ system for a later study. Instead, we will ultimately expand the Positive P function as a power series in $\epsilon$ in Section 5 .

\section{Quantum steady state}

In the previous section we described the fixed point bifurcations of the semi-classical system. Here, we investigate whether there is a signature of those semi-classical bifurcations present in the full quantum system. This can be done exactly using the positive P function, or numerically by computing the quantum steady state density operator in a truncated number basis and then constructing a phase space quasi probability density (e.g. a $Q$ function) in different regions of the semi-classical 'phase diagram' of Figure 3. As we will show, by changing the coupling parameters so as to be on different sides of a semi-classical bifurcation, there is a corresponding qualitative change in the quantum steady state. This kind of correspondence principle has proven to be the case for other dissipative nonlinear quantum systems [19-21].

\subsection{Steady state via the positive $P$ function}

The steady state solution of (8) can be found as the potential conditions are satisfied [22]. The steady state solution can be written as

$$
P_{S}(\alpha, \beta)=\mathcal{N} e^{-V(\alpha, \beta)},
$$

where the potential function is given by

$$
\begin{aligned}
V(\alpha, \beta)= & -2 \alpha \beta-\lambda \ln \left(\chi \alpha^{2}+\kappa\right)-\lambda^{*} \ln \left(\chi \beta^{2}+\kappa\right) \\
& -\frac{2 \epsilon}{\sqrt{\chi^{\kappa}}} \arctan \frac{\alpha}{A_{0}}-\frac{2 \epsilon^{*}}{\sqrt{\chi^{\kappa}}} \arctan \frac{\beta}{A_{0}^{*}},
\end{aligned}
$$


where

$$
\lambda=-1+\frac{\Delta}{\chi}-\mathrm{i} \frac{\gamma}{\chi}
$$

and $A_{0}=\sqrt{-\alpha_{0}^{2}}=\sqrt{\frac{\kappa}{\chi}}$ determines the semi-classical fixed points of the corresponding Hamiltonian (non-dissipative) model, see (18). This may be written in an alternate form by noting that $\arctan x=i \frac{1}{2} \ln \frac{1-\mathrm{i} x}{1+\mathrm{i} x}$,

$$
P_{s}(\alpha, \beta)=\mathcal{N}\left(\frac{\alpha_{0}-\alpha}{\alpha_{0}+\alpha}\right)^{\mu}\left(\frac{\alpha_{0}^{*}-\beta}{\alpha_{0}^{*}+\beta}\right)^{\mu^{*}}\left(\chi \alpha^{2}+\kappa\right)^{\lambda}\left(\chi \beta^{2}+\kappa\right)^{\lambda^{*}} \mathrm{e}^{2 \alpha \beta}
$$

where $\mu=\mathrm{i} \frac{\epsilon}{\sqrt{x \kappa}}$.

Before we can compare this distribution to the phase space structure of the semiclassical fixed points we must face the unusual feature that the Positive P function has support on a phase space with twice as many dimensions as the corresponding classical problem. The semi-classical subspace corresponds to $\beta=\alpha^{*}$. If it were not for the noise terms in the stochastic differential equations, (9), we could start on this subspace and never leave it. The noise however will drive the dynamics off the semi-classical subspace. Despite this we can find a very close correspondence between the semi-classical fixed points and the form of the steady state Positive P function.

We first discuss the correspondence for the case of no coherent driving, $\epsilon=0$. The peaks of the steady state positive $\mathrm{P}$ function will be located at the minimum of the corresponding potential function, that is to say, the solutions of, $\partial_{\alpha} V=\partial_{\beta} V=0$. This gives

$$
\begin{aligned}
& \beta=-\frac{\lambda \alpha}{\alpha^{2}-\alpha_{0}^{2}}, \\
& \alpha=-\frac{\lambda^{*} \beta}{\beta^{2}-\alpha_{0}^{* 2}},
\end{aligned}
$$

where we have used (18). A little algebra shows that these are equivalent to

$$
\begin{aligned}
& \left(\beta^{2}-\alpha_{0}^{* 2}\right)\left(\alpha^{2}-\alpha_{0}^{2}\right)=|\lambda|^{2}, \\
& \frac{1-\left(\frac{\alpha_{0}}{\alpha}\right)^{2}}{1-\left(\frac{\alpha_{0}^{*}}{\beta}\right)^{2}}=\frac{\lambda}{\lambda^{*}} .
\end{aligned}
$$

There are two classes of solutions: $\beta=\alpha^{*}$ and $\beta=-\alpha^{*}$. We will refer to the first of these as the semi-classical subspace and the second as the nonclassical.

We first consider the semi-classical subspace. With $\beta=\alpha^{*}$, the first equation in (27) should be compared with the semi-classical steady state from (11), which may be written as

$$
\alpha^{*}=-\frac{\left(\frac{\Delta}{\chi}-\mathrm{i} \frac{\gamma}{\chi}\right) \alpha}{\alpha^{2}+\frac{\kappa}{\chi}} .
$$

In the limit of small quantum noise, $\chi \rightarrow 0, \kappa \rightarrow 0$, such that $\frac{\kappa}{\chi}=$ constant we find that

$$
\lambda \approx \frac{\Delta}{\chi}-\mathrm{i} \frac{\gamma}{\chi},
$$


and, in the semi-classical subspace, the P-function is peaked on the semi-classical steady states.

In the model of Wolinsky and Carmichael [23] the nonlinear detuning $\chi$ becomes complex, thus describing nonlinear damping, and the dynamics of the positive P-function takes a very similar form to that considered here. In particular the additional fixed points of the non classical dimension are also present. As they describe, the non classical subspace allows the noise to drive a stochastic process that corresponds to the nonclassical features of the steady state solution. In the case of strong nonlinearity they show that the steady state positive P-function on the non classical subspace is localised on the non classical fixed points and that these peaks reflect the fact that the steady state is close to a superposition of two coherent states localised on the classical fixed points.

The explicit solutions to (27) are not straightforward; they are

$$
\begin{aligned}
& (\alpha, \beta)=(0,0), \\
& \left( \pm_{1} \frac{1}{\alpha_{0}^{*}} \sqrt{\left|\alpha_{0}\right|^{4}+\mathrm{i} \lambda \Im\{\lambda\} \pm_{2} \lambda \sqrt{\left|\alpha_{0}\right|^{4}-\Im\{\lambda\}^{2}}}\right. \\
& \left.\mp_{2} \pm_{1} \frac{1}{\alpha_{0}} \sqrt{\left|\alpha_{0}\right|^{4}-\mathrm{i} \lambda * \Im\{\lambda\} \pm_{2} \lambda^{*} \sqrt{\left|\alpha_{0}\right|^{4}-\Im\{\lambda\}^{2}}}\right) .
\end{aligned}
$$

These are very close, though not exactly coincident, with the semi-classical fixed points derived in Section 3.

\subsection{Numerical steady state}

To perform the numerical computation of the quantum steady state we use the Quantum Optics MATLAB toolbox [24]. To do this we approximate the infinite basis of the microwave cavity oscillator; we choose to do this by truncating in the Fock (number) basis. This means that we must choose couplings such that the bifurcation takes place sufficiently close to the origin to be accurately approximated by the truncation. This is roughly because a coherent state of amplitude $\alpha$ has a mean occupation number of $|\alpha|^{2}$. Given the quantum steady state typically (as we shall see direct evidence of in this section) has support centred on the semi-classical steady state, fixed points far from the origin (high $|\alpha|)$ will produce high occupations and thus inaccurate results if we truncate in the Fock (number) basis.

We choose a point in parameter space from each region of the semi-classical 'phase diagram' of Figure 3. These choices are marked with the black circle markers in that figure. Semi-classically, the corresponding steady states were shown in Figure 4. We now look at the quantum steady state through the Wigner function of the steady state density matrix. The Wigner function is defined as $W(x, y)=\frac{1}{\pi \hbar} \int_{-\infty}^{\infty} \mathrm{d} z\langle x-z|\hat{\rho}| x+z\rangle \mathrm{e}^{\mathrm{i} \frac{2 y z}{\hbar}}$; for more on the Wigner function see $[15,25]$. These Wigner functions are shown in Figure 6. There are clear signature of the semi-classical bifurcations. The quantum steady state has support centred on the stable semi-classical fixed points, something which has been previously observed in [19-21].

However, in two of the Wigner functions of Figure 6 (those corresponding to the striped blue region of Figure 3) there are three semi-classical stable fixed points, yet only two main regions of quantum steady state density. To investigate this further, we consider the 

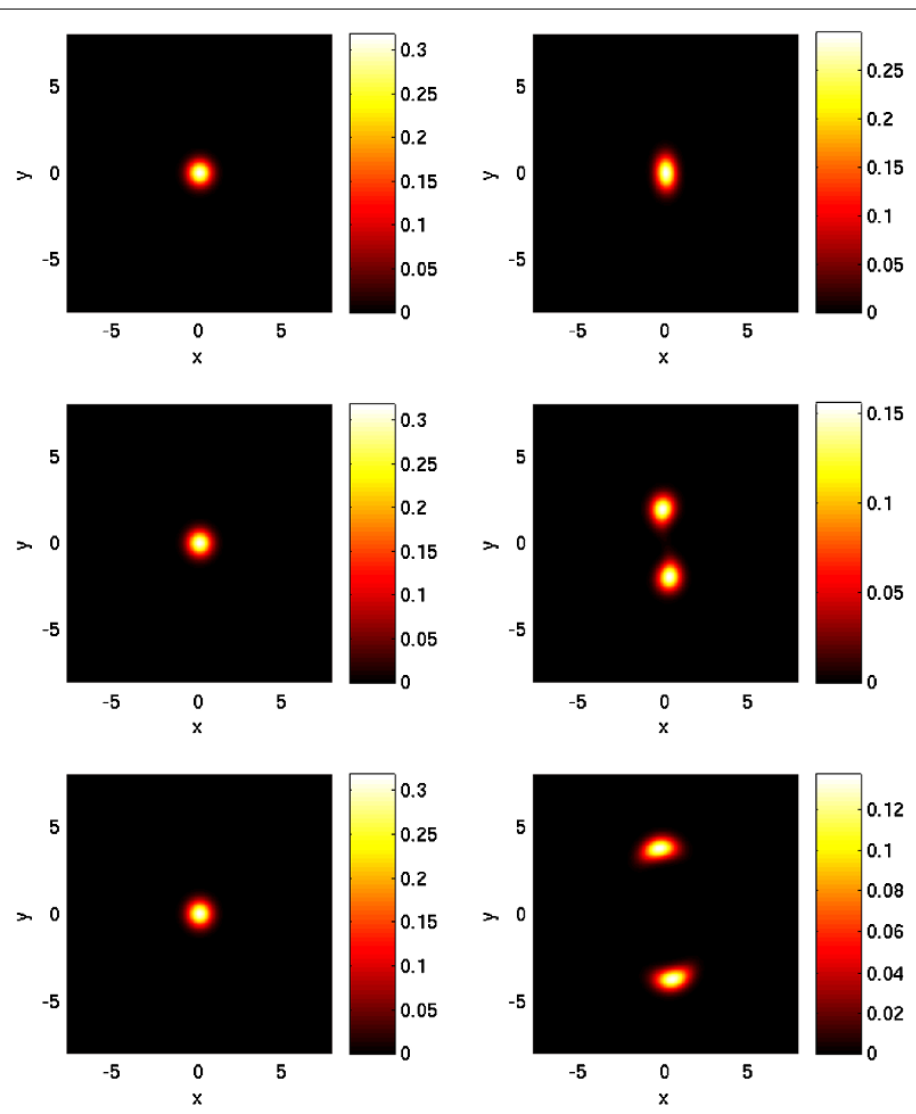

Figure 6 Steady state Wigner functions of the quantum microwave system. Density plots of steady state Wigner functions of the quantum microwave system for various parameter regimes. The Wigner function $W(x, y)$ is plotted where $x$ and $y$ are two quadratures of the microwave field. These nine plots show the quantum steady states that correspond to the parameters of the nine black circles in Figure 3. Specifically, from top-left to bottom-right, moving form left to right, then top to bottom, these parameter values are: $\left(\kappa^{\prime}, \Delta^{\prime}\right)=(0,10),(5,10),(0,0),(5,0),(0,-10)$, and $(5,-10)$. The other parameters are set to unity $\chi=\gamma=1$ for the purpose of having a Wigner density well inside the number basis truncation. The quantum steady state shows clear signs of the semi-classical bifurcations it undergoes. Particular comparison can be made to the semi-classical steady states of Figure 4 . The quantum steady state has support centred on the semi-classical stable fixed points.

quantum steady states corresponding to small parameter changes in this region. In particular, we look at the quantum steady states corresponding to the parameter space choices marked with black crosses in Figure 3. Semi-classically, the corresponding steady states were shown in Figure 5. The corresponding Wigner functions are shown in Figure 7. Interestingly, there is a gradual transition from quantum steady state support centred on the semi-classical stable fixed point at the origin, to support centred on the separated stable pair. This transition does not correspond to any semi-classical bifurcation, and at this stage is a quantum feature we cannot explain or predict semi-classically. We mention it here to suggest one direction for future investigation of this system.

\section{The small signal gain}

The positive P-function directly determines the normally ordered steady state moments of the intracavity field. We now need to chose the contour of integration so that the nor- 


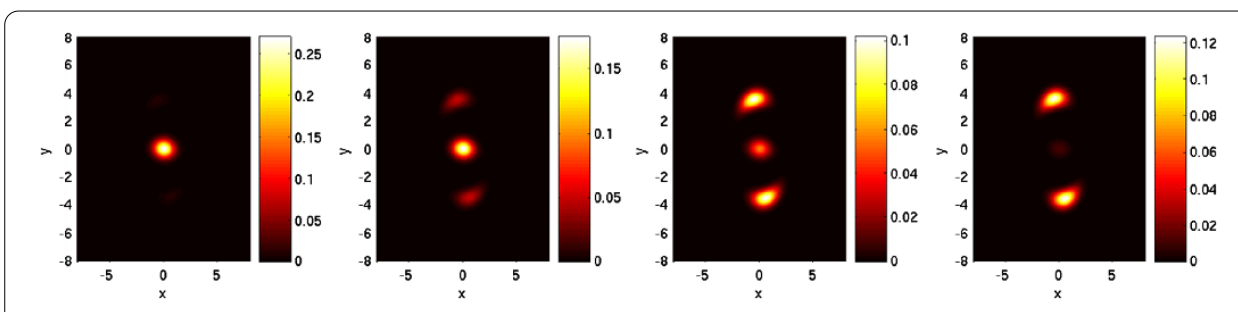

Figure 7 Steady state Wigner functions of the quantum microwave system. Density plots of steady state Wigner functions of the quantum microwave system for small parameter changes in the blue parameter region of Figure 2. The Wigner function $W(x, y)$ is plotted where $x$ and $y$ are two quadratures of the microwave field. These eight plots show the quantum steady states that correspond to the parameters of the eight black crosses in Figure 3. Specifically from left to right these parameter values are:

$\left(\kappa^{\prime}, \Delta^{\prime}\right)=(3,-10),(3.25,-10),(3.5,-10)$, and $(3.75,-10)$. The other parameters are set to unity $\chi=\gamma=1$ for the purpose of having a Wigner density well inside the number basis truncation. Comparison should be made with the semi-classical steady states of Figure 5. The quantum steady state shows support that shifts from being centred on the semi-classical stable fixed point at the origin, to being centred on the separated stable pair. This transition does not correspond to any semi-classical bifurcation. While the semi-classical steady states of Figure 5 are quite insensitive to small parameter shifts in regions bounded by semi-classical bifurcations, the corresponding quantum steady states have a marked qualitative change.

malization constant $\mathcal{N}$ is fixed. To this end we define the integrals

$$
A_{m n}=\mathcal{N}^{-1} \int \mathrm{d} \alpha \mathrm{d} \beta \alpha^{n} \beta^{m} P_{s}(\alpha, \beta),
$$

and express the normally ordered moments as

$$
\left\langle\hat{a}^{\dagger m} \hat{a}^{n}\right\rangle=\frac{A_{m n}}{A_{00}} .
$$

If we wish to regard this system as an amplifier, we need to calculate the mean cavity field amplitude $\langle\hat{a}\rangle$ as a function of $\epsilon$ for the case that $\epsilon \ll \kappa$. With this in mind we expand the solution in a Taylor series in $\epsilon$

$$
\begin{aligned}
P_{s}(\alpha, \beta)= & P_{s}^{(0)}(\alpha, \beta)-2 \mu \sum_{k=0}^{\infty} \frac{1}{2 k+1}\left(\frac{\alpha}{\alpha_{0}}\right)^{2 k+1} P_{s}^{(0)}(\alpha, \beta) \\
& -2 \mu^{*} \sum_{k=0}^{\infty} \frac{1}{2 k+1}\left(\frac{\beta}{\alpha_{0}^{*}}\right)^{2 k+1} P_{s}^{(0)}(\alpha, \beta),
\end{aligned}
$$

where $P_{s}^{(0)}(\alpha, \beta)$ is the exact steady state solution for $\epsilon=0$. Then

$$
\begin{aligned}
A_{m n}= & A_{m n}^{(0)}-2 \mu \sum_{k=0}^{\infty} \frac{1}{2 k+1}\left(\frac{\alpha}{\alpha_{0}}\right)^{2 k+1} A_{m, n+2 k+1}^{(0)} \\
& -2 \mu^{*} \sum_{k=0}^{\infty} \frac{1}{2 k+1}\left(\frac{\beta}{\alpha_{0}^{*}}\right)^{2 k+1} A_{m+2 k+1, n}^{(0)}
\end{aligned}
$$

In this form we can see that the normalisation for $P_{s}(\alpha, \beta)$ is the same as that for $P_{s}^{(0)}(\alpha, \beta)$ as the integrals $A_{0,2 k+1}^{(0)}$ vanish. 
If we now substitute (26) (with $\epsilon=0 \Rightarrow \mu=0$ ) into (33), and use the Beta function identity

$$
\left(1-\mathrm{e}^{\mathrm{i} 2 \pi \alpha}\right)\left(1-\mathrm{e}^{\mathrm{i} 2 \pi \beta}\right) \mathrm{B}(\alpha, \beta)=\int_{C} t^{\alpha-1}(1-t)^{\beta-1} \mathrm{~d} t,
$$

then we obtain the moments for zero coherent driving

$$
\begin{aligned}
A_{m n}^{(0)}= & \frac{\kappa^{\lambda+\lambda^{*}+1}\left(1-\mathrm{e}^{\mathrm{i} 2 \pi(\lambda+1)}\right)\left(1-\mathrm{e}^{\mathrm{i} 2 \pi\left(\lambda^{*}+1\right)}\right)}{-4 \chi}\left(-\frac{\kappa}{\chi}\right)^{(n+m) / 2} \\
& \times \sum_{l=0}^{\infty}\left\{\frac{1}{l !}\left(\frac{2 \kappa}{\chi}\right)^{l}\left[1+(-1)^{l+n}\right]\left[1+(-1)^{l+m}\right]\right. \\
& \left.\times \mathrm{B}\left(\lambda+1, \frac{l+n+1}{2}\right) \mathrm{B}\left(\lambda^{*}+1, \frac{l+m+1}{2}\right)\right\} .
\end{aligned}
$$

Since we will always be interested in ratios of these, we can omit the leading constant; this then exactly matches the expression found by Kryuchkyan and Kheruntsyan [26]

$$
\begin{aligned}
A_{m n}^{(0)}= & \left(-\frac{\kappa}{\chi}\right)^{(n+m) / 2} \sum_{l=0}^{\infty}\left\{\frac{1}{l !}\left(\frac{2 \kappa}{\chi}\right)^{l}\left[1+(-1)^{l+n}\right]\left[1+(-1)^{l+m}\right]\right. \\
& \left.\times \mathrm{B}\left(\lambda+1, \frac{l+n+1}{2}\right) \mathrm{B}\left(\lambda^{*}+1, \frac{l+m+1}{2}\right)\right\} .
\end{aligned}
$$

We first consider the steady state mean intra-cavity photon number with no coherent signal,

$$
\left\langle\left.\hat{a}^{\dagger} \hat{a}\right|^{(0)}=\frac{A_{11}^{(0)}}{A_{00}^{(0)}} .\right.
$$

In Figure 8 we plot this as a function of the parametric driving strength. Note that we do not see a bistable curve as in Figure 2. The reason for this is that the quantum steady state gives a long time average which averages over all possible switching events between the two semi-classical steady states in the bistable region. The quantum steady state is a double peaked distribution in the complex P representation with each peak localised near one or the other semi-classical fixed points in the bistable region.

Figure 8 The steady state mean photon number in the cavity for no linear driving. The steady state mean photon number in the cavity for no coherent driving $\epsilon$ $=0$ as a function of the parametric pump magnitude $\kappa$ and the detuning $\Delta$. The corresponding semi-classical fixed points plotted in Figure 2 showed bi-stability for negative detuning $\Delta<0$ which does not occur in the quantum steady state. Time units are chosen so that $\gamma$ $=1$ and $\chi=0.25$.

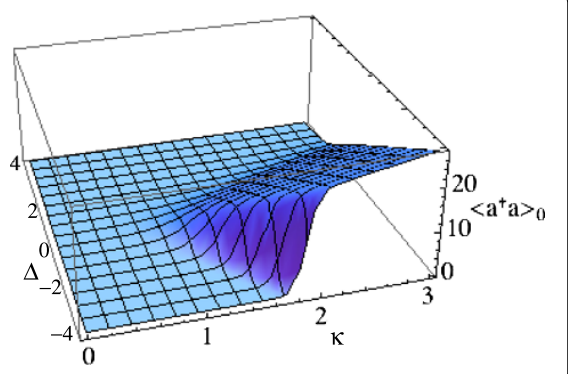


We can now write the moments of the intra-cavity field as,

$$
\begin{aligned}
\left\langle\hat{a}^{\dagger m} \hat{a}^{n}\right\rangle= & \left\langle\hat{a}^{\dagger m} \hat{a}^{n}\right\rangle^{(0)}-2 \mu \sum_{k=0}^{\infty} \frac{1}{2 k+1}\left(\frac{1}{\alpha_{0}}\right)^{2 k+1}\left\langle\hat{a}^{\dagger} \hat{a}^{n+2 k+1}\right\rangle^{(0)} \\
& -2 \mu^{*} \sum_{k=0}^{\infty} \frac{1}{2 k+1}\left(\frac{1}{\alpha_{0}^{*}}\right)^{2 k+1}\left\langle\hat{a}^{\dagger m+2 k+1} \hat{a}^{n}\right\rangle^{(0)},
\end{aligned}
$$

where $\langle\cdots\rangle_{0}$ denotes the steady state average for $\epsilon=0$. In particular, the average amplitude in the cavity at steady state is

$$
\begin{aligned}
\langle\hat{a}\rangle= & \langle\hat{a}\rangle^{(0)}-2 \mu \sum_{k=0}^{\infty} \frac{1}{2 k+1}\left(\frac{1}{\alpha_{0}}\right)^{2 k+1}\left\langle\hat{a}^{2 k+2}\right\rangle^{(0)} \\
& -2 \mu^{*} \sum_{k=0}^{\infty} \frac{1}{2 k+1}\left(\frac{1}{\alpha_{0}^{*}}\right)^{2 k+1}\left\langle\hat{a}^{\dagger}{ }^{2 k+1} \hat{a}\right\rangle^{(0)}
\end{aligned}
$$

where we have used $\langle\hat{a}\rangle_{0}=0$. Explicitly, this average amplitude is

$$
\begin{aligned}
\langle\hat{a}\rangle= & -2 \frac{\alpha_{0}}{N} \sum_{k=0}^{\infty} \sum_{r=0}^{\infty} \frac{1}{2 k+1} \frac{2^{2 r}\left|\alpha_{0}\right|^{4 r}}{(2 r) !} \Gamma\left(\frac{1}{2}+r\right) \Gamma\left(\frac{3}{2}+r+k\right) \\
& \times\left[\frac{\mu}{\Gamma\left(\frac{3}{2}+r+\lambda^{*}\right) \Gamma\left(\frac{5}{2}+r+k+\lambda\right)}+\frac{\mu^{*}\left|\alpha_{0}\right|^{2}}{\Gamma\left(\frac{5}{2}+r+\lambda\right) \Gamma\left(\frac{5}{2}+r+k+\lambda^{*}\right)}\right],
\end{aligned}
$$

where

$$
N=\sum_{s=0}^{\infty} \frac{2^{2 s}\left|\alpha_{0}\right|^{4 s}}{(2 s) !} \frac{\left|\Gamma\left(\frac{1}{2}+s\right)\right|^{2}}{\left|\Gamma\left(\frac{3}{2}+s+\lambda\right)\right|^{2}}
$$

we recall that $\alpha_{0}^{2}=-\frac{\kappa}{\chi}, \lambda=-1+\frac{\Delta}{\chi}-\mathrm{i} \frac{\gamma}{\chi}$, and $\mu=\mathrm{i} \frac{\epsilon}{\sqrt{\chi \kappa}}=\mathrm{i} \frac{1}{\sqrt{\frac{\kappa}{\chi}}} \frac{\epsilon}{|\chi|}$. Writing $\epsilon=|\epsilon| \mathrm{e}^{\mathrm{i} v}$, we can obtain the magnitude of the cavity field at steady state $|\langle\hat{a}\rangle|$,

$$
|\langle\hat{a}\rangle|=G\left(\frac{\kappa}{\chi}, \frac{\Delta}{\chi}, \frac{\gamma}{\chi}, v\right)\left|\frac{\epsilon}{\chi}\right|,
$$

where the gain $G=G\left(\frac{\kappa}{\chi}, \frac{\Delta}{\chi}, \frac{\gamma}{\chi}, v\right) \geq 0$ is

$$
\begin{aligned}
G= & \frac{2}{R} \mid \sum_{k=0}^{\infty} \sum_{r=0}^{\infty} \frac{1}{2 k+1} \frac{2^{2 r}}{(2 r) !}\left(\frac{\kappa}{\chi}\right)^{2 r} \Gamma\left(\frac{1}{2}+r\right) \Gamma\left(\frac{3}{2}+r+k\right) \\
& \times\left[\frac{1}{S}-\frac{\left(\frac{\kappa}{\chi}\right) \mathrm{e}^{-2 \mathrm{i} v}}{S^{*}}\right] \mid,
\end{aligned}
$$

where

$$
\begin{aligned}
& R=\sum_{s=0}^{\infty} \frac{2^{2 s}}{(2 s) !}\left(\frac{\kappa}{\chi}\right)^{2 s} \frac{\left|\Gamma\left(\frac{1}{2}+s\right)\right|^{2}}{\left|\Gamma\left(\frac{1}{2}+s+\frac{\Delta}{\chi}-\mathrm{i} \frac{\gamma}{\chi}\right)\right|^{2}}, \\
& S=\Gamma\left(\frac{1}{2}+r+\frac{\Delta}{\chi}+\mathrm{i} \frac{\gamma}{\chi}\right) \Gamma\left(\frac{3}{2}+r+k+\frac{\Delta}{\chi}-\mathrm{i} \frac{\gamma}{\chi}\right) .
\end{aligned}
$$




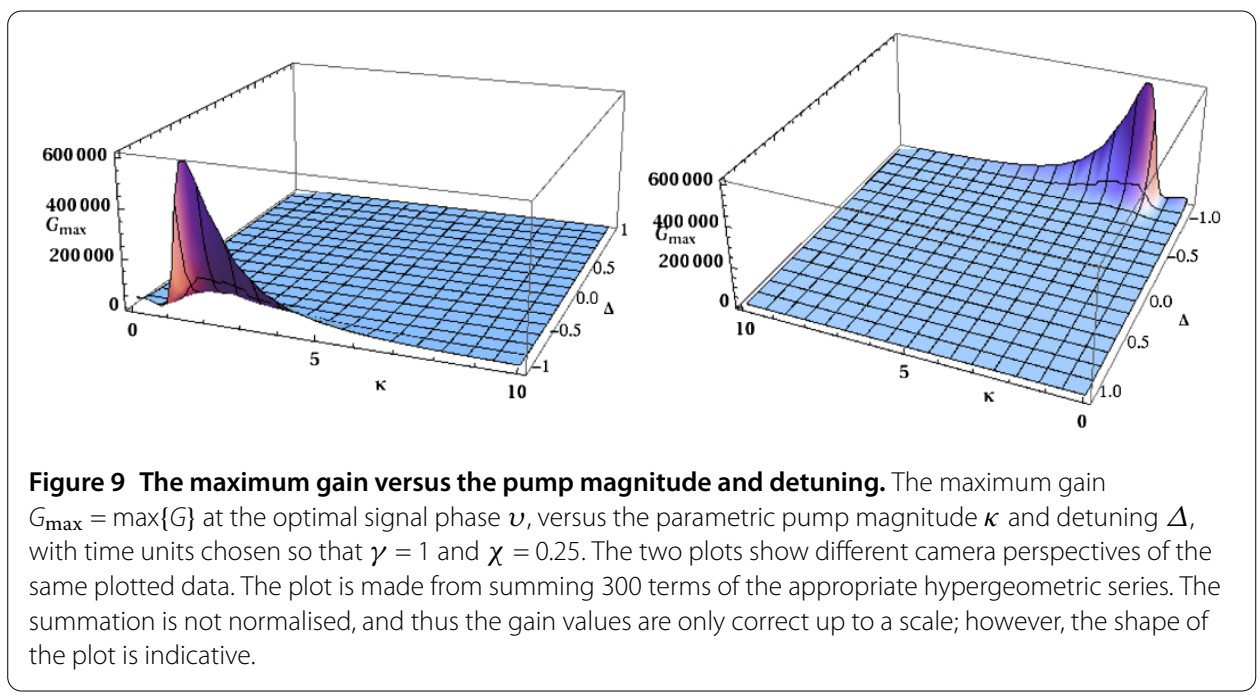

Figure 10 The superconducting microwave resonator modelled as a single-sided cavity for use as an input-output formulation.

The superconducting microwave resonator modelled as a single-sided cavity for use as an input-output formulation. The incoming field mode operator is $\hat{a}_{i}(t)$ and the outgoing field mode operator is $\hat{a}_{o}(t)$. Loss from the microwave cavity occurs at a rate $\gamma$. Note here that $\gamma$ is

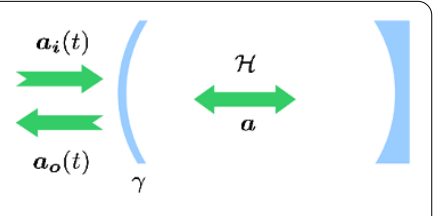
the coefficient of the amplitude decay, the coefficient for the photon

number loss is $2 \gamma$. The Hamiltonian dynamics of the cavity mode $\hat{a}$ are governed by the Interaction picture Hamiltonian $\mathcal{H}$.

In Figure 9 we plot the maximum gain $G_{\max }=\max \{G\}$ for a given parametric pump strength $\kappa$ and detuning $\Delta$. We have plotted the maximum gain by choosing the optimal signal phase $v$ at each set of parameters. Comparing this to Figure 8 for the case when there is no coherent driving, we see that the gain is a maximum around the critical parametric driving strength in the bi-stable, negatively detuned region.

\section{Linearised quantum system}

\subsection{Input-output formalism}

We consider the microwave cavity with the input-output formulation of quantum optics, as originally described by Collett and Gardiner in [27]. To do this, we model the superconducting microwave resonator as a single-sided cavity as depicted in Figure 10.

The input and output fields are treated explicitly with their mode annihilation operators $\hat{a}_{i}$ and $\hat{a}_{o}$ respectively. With this formulation, the quantum stochastic differential equation we obtain for the microwave resonator field mode operators $\hat{a}$ and $\hat{a}^{\dagger}$ are

$$
\begin{aligned}
& \frac{\mathrm{d} \hat{a}}{\mathrm{~d} t}=-\frac{\mathrm{i}}{\hbar}[\hat{a}, \mathcal{H}]-\gamma \hat{a}+\sqrt{2 \gamma} \hat{a}_{i}(t), \\
& \frac{\mathrm{d} \hat{a}^{\dagger}}{\mathrm{d} t}=-\frac{\mathrm{i}}{\hbar}\left[\hat{a}^{\dagger}, \mathcal{H}\right]-\gamma \hat{a}^{\dagger}+\sqrt{2 \gamma} \hat{a}_{i}^{\dagger}(t),
\end{aligned}
$$

where the input field is effectively white noise, uncorrelated in time,

$$
\left[\hat{a}_{i}(t), \hat{a}_{i}^{\dagger}\left(t^{\prime}\right)\right]=\delta\left(t-t^{\prime}\right) \hat{I}
$$


The probability per unit time to detect a photon in the input field is $2 \gamma\left\langle\hat{a}_{i}^{\dagger}(t) \hat{a}_{i}(t)\right\rangle$. Finally, the relationship between the input, output, and cavity fields is given by

$$
\hat{a}_{o}(t)=\sqrt{2 \gamma} \hat{a}(t)+\mathrm{e}^{\mathrm{i} \xi} \hat{a}_{i}(t)
$$

where the phase of the second term, the reflected input, may vary with the system. For an almost perfectly reflecting mirror of an optical cavity we have $\xi=\pi$ and $\mathrm{e}^{\mathrm{i} \xi}=-1$, here we choose this phase as an appropriate approximation.

We also look at the various fields in the frequency domain, by defining the frequencydomain operators as the time-domain operators' Fourier transforms,

$$
\begin{aligned}
& \hat{\tilde{a}}(\omega)=\mathcal{F}_{t \rightarrow \omega}\{\hat{a}(t)\}, \\
& \hat{\tilde{a}}_{i}(\omega)=\mathcal{F}_{t \rightarrow \omega}\left\{\hat{a}_{i}(t)\right\}, \\
& \hat{\tilde{a}}_{o}(\omega)=\mathcal{F}_{t \rightarrow \omega}\left\{\hat{a}_{o}(t)\right\},
\end{aligned}
$$

where we have used the Fourier Transform convention $\tilde{f}(\omega)=\mathcal{F}_{t \rightarrow \omega}\{f(t)\}=\frac{1}{\sqrt{2 \pi}} \times$ $\int_{-\infty}^{\infty} \mathrm{d} x f(t) \mathrm{e}^{-\mathrm{i} \omega t}$. In the frequency domain, the input field is also uncorrelated in frequency,

$$
\left[\hat{\tilde{a}}_{i}(\omega), \hat{\tilde{a}}_{i}^{\dagger}\left(\omega^{\prime}\right)\right]=\delta\left(\omega-\omega^{\prime}\right) \hat{I}
$$

and the relationship between the input, output, and cavity fields is then given by

$$
\hat{\tilde{a}}_{o}(\omega)=\sqrt{2 \gamma} \hat{\tilde{a}}(\omega)-\hat{\tilde{a}}_{i}(\omega) .
$$

\subsection{Gain spectra}

Recall our quantum equations of motion for the microwave cavity field (47). We linearise the system about a semi-classical fixed point $\alpha_{0}$ as we did semi-classically in (14). This gives us the linearised equation of motion for the fluctuation

$$
\left[\begin{array}{c}
\frac{\mathrm{d}(\widehat{\delta a}(t))}{\mathrm{d} t} \\
\frac{\mathrm{d}\left(\hat{\delta a}^{\dagger}(t)\right)}{\mathrm{d} t}
\end{array}\right]=\mathbf{M}_{\alpha \alpha^{*}}\left[\begin{array}{c}
\widehat{\delta a}(t) \\
\widehat{\delta a}^{\dagger}(t)
\end{array}\right]+\sqrt{2 \gamma}\left[\begin{array}{c}
\hat{a}_{i}(t) \\
\hat{a}_{i}^{\dagger}(t)
\end{array}\right],
$$

where

$$
\widehat{\delta a}(t)=\hat{a}(t)-\alpha_{0} .
$$

In the frequency domain the linearised equation of motion (53) becomes

$$
\mathrm{i} \omega\left[\begin{array}{c}
\hat{\tilde{\delta} a}(\omega) \\
\hat{\tilde{\delta}}^{\dagger}(-\omega)
\end{array}\right]=\mathbf{M}_{\alpha \alpha^{*}}\left[\begin{array}{c}
\hat{\tilde{\delta}} a(\omega) \\
\hat{\tilde{\delta}}^{\dagger}(-\omega)
\end{array}\right]+\sqrt{2 \gamma}\left[\begin{array}{c}
\hat{\tilde{a}}_{i}(\omega) \\
\hat{\tilde{a}}_{i}^{\dagger}(-\omega)
\end{array}\right] .
$$

We rewrite this to obtain an expression for the microwave cavity field fluctuation in terms of the input radiation,

$$
\left[\begin{array}{c}
\hat{\tilde{\delta} a}(\omega) \\
\hat{\tilde{\delta}}^{\dagger}(-\omega)
\end{array}\right]=\sqrt{2 \gamma}\left(\mathrm{i} \omega \mathbf{I}-\mathbf{M}_{\alpha \alpha^{*}}\right)^{-1}\left[\begin{array}{c}
\hat{\tilde{a}}_{i}(\omega) \\
\hat{\tilde{a}}_{i}^{\dagger}(-\omega)
\end{array}\right] .
$$


Using this expression, together with our input-output expression (52), allows us to obtain an expression for the output fluctuation of the microwave cavity in terms of the input,

$$
\left[\begin{array}{c}
\hat{a}_{o}(\omega) \\
\hat{\tilde{a}}_{o}^{\dagger}(-\omega)
\end{array}\right]=\mathbf{G}(\omega)\left[\begin{array}{c}
\hat{\tilde{a}}_{i}(\omega) \\
\hat{\tilde{a}}_{i}^{\dagger}(-\omega)
\end{array}\right],
$$

where the gain matrix $\mathbf{G}(\omega)$ is

$$
\mathbf{G}(\omega)=\left[\begin{array}{ll}
G_{11}(\omega) & G_{12}(\omega) \\
G_{21}(\omega) & G_{22}(\omega)
\end{array}\right]=2 \gamma\left(\mathrm{i} \omega \mathbf{I}-\mathbf{M}_{\alpha \alpha^{*}}\right)^{-1}-\mathbf{I} .
$$

Note that $\hat{\grave{a}}_{o}(\omega)$ is the output fluctuation in the frequency domain. If we re-introduced the coherent term we would obtain the full output amplitude in the frequency domain, $\hat{\tilde{a}}_{o}(\omega)=\hat{\tilde{a}}_{o}(\omega)+\sqrt{4 \pi \gamma} \alpha_{0} \delta(\omega) \hat{I}$.

Now, we can rewrite our Jacobian $\mathbf{M}_{\alpha \alpha^{*}}$ from (15) in terms of the parameters we defined in (13) for our semi-classical steady states,

$$
\mathbf{M}_{\alpha \alpha^{*}}=\gamma\left[\begin{array}{cc}
-1-\mathrm{i}\left(\Delta^{\prime}+2 \bar{n}_{0}\right) & -\mathrm{i} \bar{n}_{0} \mathrm{e}^{\mathrm{i} 2 \theta_{0}}-\mathrm{i} \kappa^{\prime} \\
\mathrm{i} \bar{n}_{0} \mathrm{e}^{-\mathrm{i} 2 \theta_{0}}+\mathrm{i} \kappa^{\prime} & -1+\mathrm{i}\left(\Delta^{\prime}+2 \bar{n}_{0}\right)
\end{array}\right] .
$$

We now introduce two other useful parameters, $\Lambda_{0} \in \mathbb{R}$, and $\omega^{\prime} \in \mathbb{R}$,

$$
\begin{aligned}
& \Lambda_{0}=\left(\kappa^{\prime}\right)^{2}-\Delta^{\prime 2}+\bar{n}_{0}\left(-4 \Delta^{\prime}+2 \kappa^{\prime} \cos \left(2 \theta_{0}\right)-3 \bar{n}_{0}\right), \\
& \omega^{\prime}=\frac{\omega}{\gamma} .
\end{aligned}
$$

In terms of these parameters, the gain matrix $\mathbf{G}(\omega)$ is

$$
\begin{aligned}
\mathbf{G}(\omega)= & \frac{1}{\Lambda_{0}+\left(\omega^{\prime}-\mathrm{i}\right)^{2}} \\
& \times\left[\begin{array}{cc}
-1-\Lambda_{0}-\omega^{\prime 2}+2 \mathrm{i}\left(\Delta^{\prime}+2 \bar{n}_{0}\right) & 2 \mathrm{i} \bar{n}_{0} \mathrm{e}^{\mathrm{i} 2 \theta_{0}}+2 \mathrm{i} \kappa \\
-2 \mathrm{i} \bar{n}_{0} \mathrm{e}^{-2 \mathrm{i} \theta_{0}}-2 \mathrm{i} \kappa & -1-\Lambda_{0}-\omega^{\prime 2}-2 \mathrm{i}\left(\Delta^{\prime}+2 \bar{n}_{0}\right)
\end{array}\right] .
\end{aligned}
$$

To calculate the gain measured at an arbitrary phase, we first define the quadrature operator in the frequency domain $\hat{X}_{\phi}(\omega)=\hat{\hat{a}}_{o}(\omega) \mathrm{e}^{\mathrm{i} \phi}+\hat{\hat{a}}_{o}^{\dagger}(-\omega) \mathrm{e}^{-\mathrm{i} \phi}$, which can be written in terms of our gain matrix as

$$
\hat{X}_{\phi}(\omega)=\left[\begin{array}{ll}
\mathrm{e}^{\mathrm{i} \phi} & \mathrm{e}^{-\mathrm{i} \phi}
\end{array}\right] \mathbf{G}(\omega)\left[\begin{array}{c}
\hat{\tilde{a}}_{i}(\omega) \\
\hat{\tilde{a}}_{i}^{\dagger}(-\omega)
\end{array}\right] .
$$

This expression reduces to

$$
\hat{X}_{\phi}(\omega)=g_{\phi}(\omega)\left(\hat{\tilde{a}}_{i}(\omega) \mathrm{e}^{\mathrm{i}(\phi+\zeta(\omega))}+\hat{\tilde{a}}_{i}^{\dagger}(-\omega) \mathrm{e}^{-\mathrm{i}(\phi+\zeta(\omega))}\right),
$$

where our signal gain $g_{\phi}(\omega)$ at phase $\phi$ is

$$
g_{\phi}(\omega)=\frac{\left|-1-\Lambda_{0}-\omega^{\prime 2}-2 \mathrm{ie}^{-2 \mathrm{i} \phi}\left(\kappa^{\prime}+\bar{n}_{0} \mathrm{e}^{-2 \mathrm{i} \theta_{0}}\right)+2 \mathrm{i}\left(\Delta^{\prime}+2 \bar{n}_{0}\right)\right|}{\Lambda_{0}+\left(\omega^{\prime}-\mathrm{i}\right)^{2}},
$$




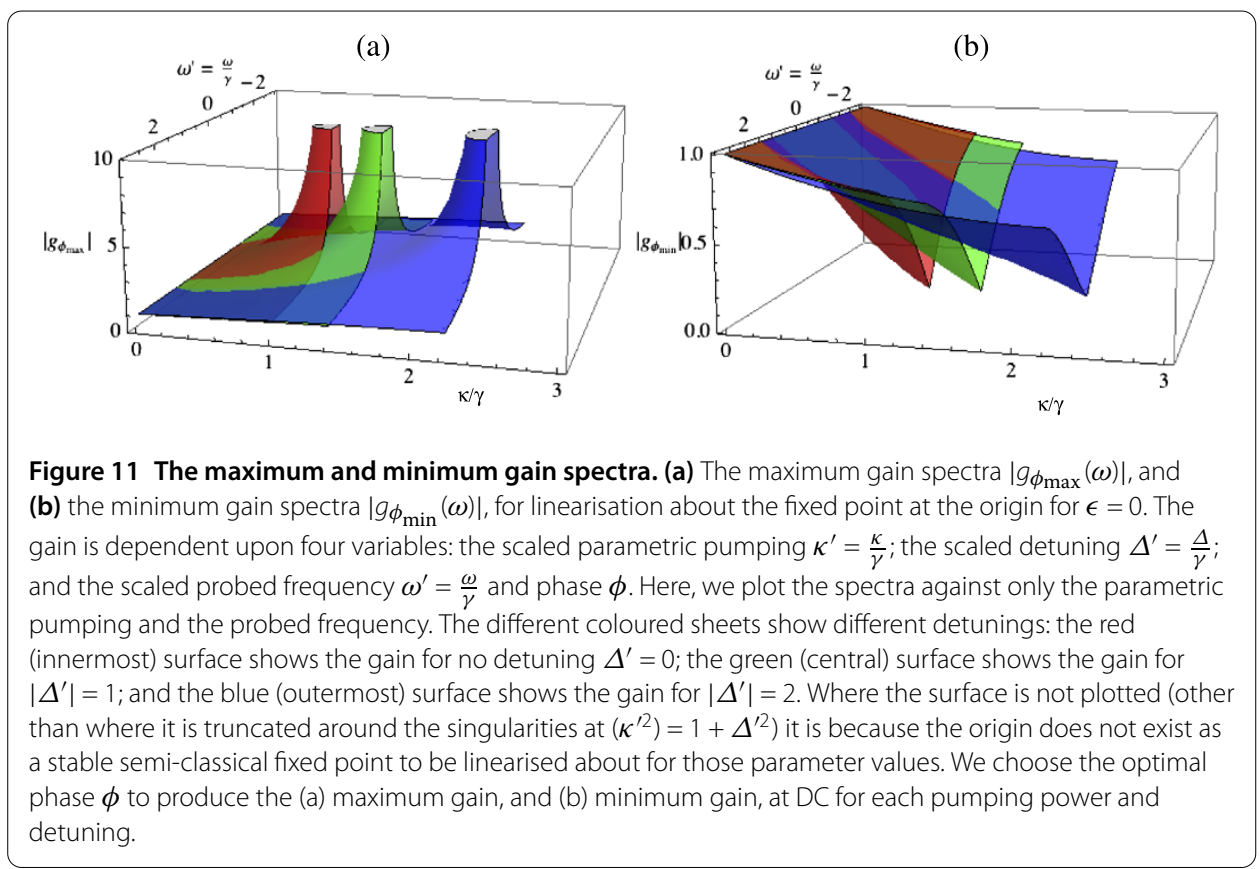

and the frequency-dependent phase shift $\zeta(\omega)$ is

$$
\zeta(\omega)=\arg \left(-1-\Lambda_{0}-\omega^{\prime 2}-2 \mathrm{ie}^{-2 \mathrm{i} \phi}\left(\kappa^{\prime}+\bar{n}_{0} \mathrm{e}^{-2 \mathrm{i} \theta_{0}}\right)+2 \mathrm{i}\left(\Delta^{\prime}+2 \bar{n}_{0}\right)\right) .
$$

Note that while our signal gain $g_{\phi}(\omega)$ is complex for non-zero frequency, it is real for the DC frequency in this frame.

If we consider our analytically solved case of no linear driving bias $\left(\epsilon=\bar{\epsilon}^{\prime}=0\right)$, and linearise about the 'below threshold' stable fixed point at the origin, then $\Lambda_{0}=\kappa^{\prime 2}-\Delta^{\prime 2}<1$ and our gain $\left|g_{\phi}(\omega)\right|$ at a phase $\phi$ can be plotted against the scaled parametric pumping $\kappa^{\prime}$ and detuning $\Delta^{\prime}$. We plot the maximum gain $\left|g_{\phi_{\max }}(\omega)\right|$ for each value of parametric pumping and detuning (optimising $\phi$ to find the maximum gain at DC for each pair of these parameters) in Figure 11.

\subsection{Squeezing spectra}

Having derived the gain matrix (61) relating the input to the output, we are now also in a position to investigate the squeezing spectrum of the microwave system. Recall our quadrature operator in the frequency domain $\hat{X}_{\phi}(\omega)=\hat{\grave{a}}_{o}(\omega) \mathrm{e}^{\mathrm{i} \phi}+\hat{\grave{a}}_{o}^{\dagger}(-\omega) \mathrm{e}^{-\mathrm{i} \phi}$ written in terms of our gain matrix in (62). Our squeezing spectrum is the variance of this quadrature operator. We thus define this squeezing spectrum $S_{\phi}(\omega)$, again in the frequency domain, to be

$$
S_{\phi}(\omega)=\int_{-\infty}^{\infty}\left\langle\hat{X}_{\phi}(\omega), \hat{X}_{\phi}\left(\omega^{\prime}\right)\right\rangle \mathrm{d} \omega^{\prime}
$$

where the notation for the covariance bracket is $\langle\hat{A}, \hat{B}\rangle=\langle\hat{A} \hat{B}\rangle-\langle\hat{A}\rangle\langle\hat{B}\rangle$. We then use the linearity of both arguments of the covariance bracket to express the variance of the quadra- 
ture operator as

$$
\begin{aligned}
S_{\phi}(\omega)= & \int_{-\infty}^{\infty}\left[\begin{array}{ll}
\mathrm{e}^{\mathrm{i} \phi} & \mathrm{e}^{-\mathrm{i} \phi}
\end{array}\right] \mathbf{G}(\omega)\left[\begin{array}{cc}
\left\langle\hat{\tilde{a}}_{i}(\omega), \hat{\tilde{a}}_{i}\left(\omega^{\prime}\right)\right\rangle & \left\langle\hat{\tilde{a}}_{i}(\omega), \hat{\tilde{a}}_{i}^{\dagger}\left(-\omega^{\prime}\right)\right\rangle \\
\left\langle\hat{\tilde{a}}_{i}^{\dagger}(-\omega), \hat{\tilde{a}}_{i}\left(\omega^{\prime}\right)\right\rangle & \left\langle\hat{\tilde{a}}_{i}^{\dagger}(-\omega), \hat{\tilde{a}}_{i}^{\dagger}\left(-\omega^{\prime}\right)\right\rangle
\end{array}\right] \\
& \times \mathbf{G}\left(\omega^{\prime}\right)^{T}\left[\begin{array}{c}
\mathrm{e}^{\mathrm{i} \phi} \\
\mathrm{e}^{-\mathrm{i} \phi}
\end{array}\right] \mathrm{d} \omega^{\prime} .
\end{aligned}
$$

Then, using the commutation relation of (51) we can rewrite $S_{\phi}(\omega)$ in terms of normallyordered variances of the input field as

$$
\begin{aligned}
& S_{\phi}(\omega)=\int_{-\infty}^{\infty}\left[\begin{array}{ll}
\mathrm{e}^{\mathrm{i} \phi} & \mathrm{e}^{-\mathrm{i} \phi}
\end{array}\right] \mathbf{G}(\omega)\left[\begin{array}{cc}
\left\langle\hat{\tilde{a}}_{i}(\omega), \hat{\tilde{a}}_{i}\left(\omega^{\prime}\right)\right\rangle & \left\langle\hat{\tilde{a}}_{i}^{\dagger}(-\omega), \hat{\tilde{a}}_{i}\left(\omega^{\prime}\right)\right\rangle \\
\left\langle\hat{\tilde{a}}_{i}^{\dagger}(-\omega), \hat{\tilde{a}}_{i}\left(\omega^{\prime}\right)\right\rangle & \left\langle\hat{\tilde{a}}_{i}^{\dagger}(-\omega), \hat{\tilde{a}}_{i}^{\dagger}\left(-\omega^{\prime}\right)\right\rangle
\end{array}\right] \\
& \times \mathbf{G}\left(\omega^{\prime}\right)^{T}\left[\begin{array}{c}
\mathrm{e}^{\mathrm{i} \phi} \\
\mathrm{e}^{-\mathrm{i} \phi}
\end{array}\right] \mathrm{d} \omega^{\prime} .
\end{aligned}
$$

To proceed we now use the statistics of the input field. A coherent input field has zero normally-ordered variances $\left(\left\langle\hat{\tilde{a}}_{i}(\omega), \hat{\tilde{a}}_{i}\left(\omega^{\prime}\right)\right\rangle=\left\langle\hat{\tilde{a}}_{i}^{\dagger}\left(-\omega^{\prime}\right), \hat{\tilde{a}}_{i}(\omega)\right\rangle=\left\langle\hat{\tilde{a}}_{i}^{\dagger}(-\omega), \hat{\tilde{a}}_{i}^{\dagger}\left(-\omega^{\prime}\right)\right\rangle=\right.$ $0)$. Thus, the only non-zero term in central matrix is the delta function term arising from the commutation relations. Using this, we can compute the integral of the matrix expression, and our squeezing spectrum reduces to

$$
\begin{aligned}
S_{\phi}(\omega)= & G_{11}(\omega) G_{12}(-\omega) \mathrm{e}^{\mathrm{i} 2 \phi}+G_{11}(\omega) G_{22}(-\omega) \\
& +G_{21}(\omega) G_{12}(-\omega)+G_{21}(\omega) G_{22}(-\omega) \mathrm{e}^{-\mathrm{i} 2 \phi},
\end{aligned}
$$

or explicitly,

$$
S_{\phi}(\omega)=\frac{\left|-1-\Lambda_{0}-\omega^{\prime 2}-\mathrm{i} 2 \mathrm{e}^{-\mathrm{i} 2 \phi}\left(\kappa^{\prime}+\bar{n}_{0} \mathrm{e}^{-\mathrm{i} 2 \theta_{0}}\right)+\mathrm{i} 2\left(\Delta^{\prime}+2 \bar{n}_{0}\right)\right|^{2}}{\left|\Lambda_{0}+\left(\omega^{\prime}-\mathrm{i}\right)^{2}\right|^{2}} .
$$

If we consider our analytically solved case of no linear driving bias $\left(\epsilon=\bar{\epsilon}^{\prime}=0\right)$, and linearise about the 'below threshold' stable fixed point at the origin, then $\Lambda_{0}=\kappa^{\prime 2}-\Delta^{\prime 2}<1$ and our squeezing $S_{\phi}(\omega)$ at a phase $\phi$ can be plotted against the scaled parametric pumping $\kappa^{\prime}$ and detuning $\Delta^{\prime}$. We plot the squeezing spectrum for each value of parametric pumping and detuning (setting the phase $\phi$ to be that which gives the minimum noise searching over all frequencies for each pair of these parameters) in Figure 12.

\subsection{Signal to noise ratio}

For operation of the microwave system as a bifurcation amplifier, the parameters which result in maximum gain may not result in minimum noise. Instead, rather than optimising for maximum gain or minimum noise individually, the quantity which we wish to maximise is the signal to noise ratio. However, we see from our expressions for the gain (64) and noise (70), that $S_{\phi}(\omega)=\left|g_{\phi}(\omega)\right|^{2}$, and that our signal to noise ratio is thus unity,

$$
\operatorname{SNR}_{\phi}(\omega)=\frac{\left|g_{\phi}(\omega)\right|}{\sqrt{S_{\phi}(\omega)}}=1
$$


Figure 12 The minimum possible noise spectra. The minimum possible noise spectra $S_{\phi_{\min }}(\omega)$ for linearisation about the fixed point at the origin for $\epsilon$ $=0$. The noise spectrum is dependent on four parameters: the scaled parametric pumping $\kappa^{\prime}=\frac{\kappa}{\gamma}$; the scaled detuning $\Delta^{\prime}=\frac{\Delta}{\gamma}$; and the scaled probed frequency $\omega^{\prime}=\frac{\omega}{\gamma}$ and phase $\phi$. Here we plot the noise spectrum against only the parametric pumping and the probed frequency. The different coloured sheets show different detunings: the red (innermost) surface shows the noise for no detuning $\Delta^{\prime}=0$; the green (central) surface shows the noise

for $\left|\Delta^{\prime}\right|=1$; and the blue (outermost) surface shows the noise for $\left|\Delta^{\prime}\right|=2$. Where the surface is not plotted it is because the origin does not exist as a stable semi-classical fixed point to be linearised about for those parameter values. We choose the optimal phase $\phi$ to produce the minimum noise $S_{\phi}(\omega)$ searching over all frequencies for each pumping power and detuning.

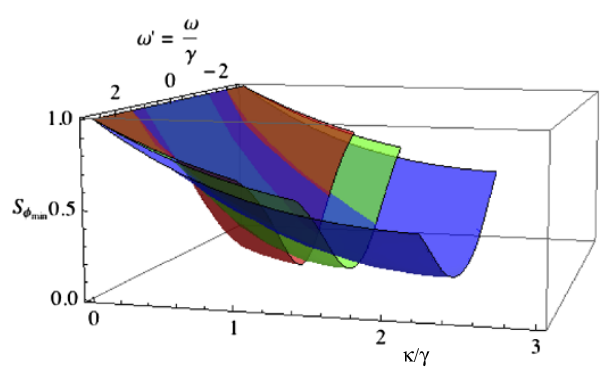

For the linearised system, this equality holds for all values of all parameters (parametric pumping, detuning, and cavity dissipation), all probed frequencies and phases, and regardless of which semi-classical fixed point we choose to linearise about.

Physically the means that our system is acting as a parametric amplifier. The quadrature of maximum gain is the same as the quadrature of maximum noise, and vice-versa for the minimum gain and noise. We can thus use this microwave system to amplify a signal to a measurable level without affecting its signal to noise ratio.

\section{Conclusion}

In this paper we detailed the quantum and semi-classical structure of a superconducting microwave resonator connected through a SQUID loop to ground. In particular we observed that the semi-classical model contains a bifurcation structure, and that the remains of this structure are still visible in the full quantum mechanical steady state. Furthermore, we showed it can be used as a bifurcation amplifier. We did this analysis by: linearising about the semi-classical steady state below the 'threshold' of the amplifier; by truncating the oscillator basis in the Fock basis and numerically computing the quantum phase space at steady state; and also by computing the exact quantum steady state by using an analytical phase space technique.

First, we showed that the corresponding semi-classical model has its fixed points determined by a quintic polynomial. We showed that for the small linear signal regime $\epsilon=0$, that this quintic factors and is analytically solvable. This semi-classical system then undergoes a bifurcation of its semi-classical steady state with increased parametric pumping power. This bifurcation gives a threshold for the amplifier and occurs when the parametric pumping power equals the cavity decay, with adjustment for a detuned drive, $|\kappa|^{2}=\gamma^{2}+\Delta^{2}$. The sign of the detuning specifies the form of the bifurcations. For a positive detuning $\Delta \geq 0$, the origin undergoes a supercritical pitchfork bifurcation at the threshold. For negative detuning $\Delta<0$, the origin instead loses its stability at $|\kappa|^{2}=\gamma^{2}+\Delta^{2}$ in a subcritical pitchfork bifurcation with two intermediate pairs of fixed points created in saddle-node bifurcations when the parametric pumping power reaches $|\kappa|^{2}=\gamma^{2}$. The numerically calculated quantum steady states were shown to have clear signatures of these semi-classical steady state bifurcations. Specifically, the Wigner function representation of the quantum phase space was seen to have support on the semi-classical fixed points. 
In addition to numerically computing the quantum phase space at steady state by truncating the oscillator basis, we also calculated the exact quantum steady state. This was done following the work of Kryuchkyan and Kheruntsyan [26] by using the Positive P representation. The method took advantage of the fact that the potential conditions were satisfied. The exact quantum phase space density at steady state was seen to be peaked in the vicinity of the corresponding semi-classical fixed points.

We showed that the quantum device functioned as a bifurcation amplifier until threshold. We calculated the small signal gain of the amplifier using the exact quantum steady state. We also approximated this by linearising the steady state about the semi-classical below-threshold fixed point using the input-output formalism of Collett and Gardiner [27]. With this procedure we also calculated noise spectra, and we showed that the signal to noise ratio at all frequencies and phases was equal to unity. We thus showed that the quarter-wave microwave resonator considered can be made to act as a parametric amplifier. This device can take a signal from a nano-electromechanical system and amplify it to a measurable level without affecting its signal to noise ratio.

Competing interests

The authors declare that they have no competing interests.

Authors' contributions

The paper was written by CPM who did the numerical simulations and, together with GJM, performed the analytic calculations. HN obtained the solution in Section 4. TD provided some of the experimental parameters.

\section{Author details}

${ }^{1}$ Department of Physics, The University of Queensland, St Lucia, QLD 4072, Australia. ${ }^{2}$ Department of Physics, Texas A \& M University at Qatar, PO Box 23874, Doha, Qatar. ${ }^{3}$ Department of Physics, The University of New South Wales, Kensington, NSW 2052, Australia.

\section{Acknowledgements}

This work was supported by the Australian Research Council grants FF0776191 and CE110001014.

Received: 29 January 2014 Accepted: 24 April 2014 Published: 10 June 2014

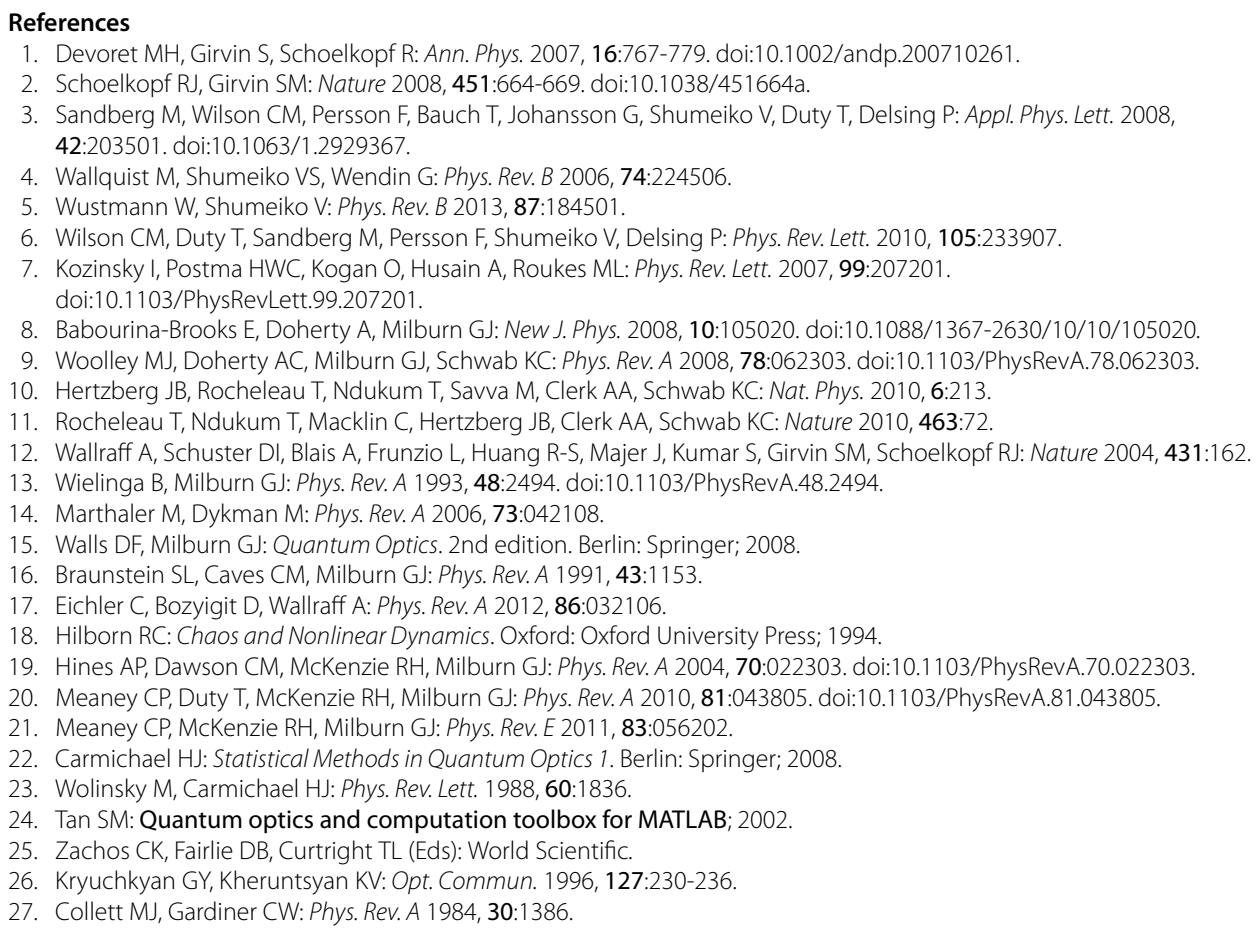


doi:10.1140/epjqt7

Cite this article as: Meaney et al.: Quantum and classical nonlinear dynamics in a microwave cavity. EPJ Quantum Technology 2014 1:7.

Submit your manuscript to a SpringerOpen ${ }^{\circ}$ journal and benefit from:

- Convenient online submission

- Rigorous peer review

- Immediate publication on acceptance

Open access: articles freely available online

- High visibility within the field

- Retaining the copyright to your article

Submit your next manuscript at $\boldsymbol{s p r i n g e r o p e n . c o m ~}$ 